\title{
Obsessive-Compulsive Disorder in Hospitalized Patients with Schizophrenia
}

\author{
Mercedeh Samiei ${ }^{1}$, Koorosh Hedayati $^{2}$, Arash Mirabzadeh Ardekani ${ }^{1}$, Behrooz Dolatshahi $^{3}$, Reza Daneshmand $^{3^{*}}$, Roya Samadi $^{4}$ \\ 1. Department of Psychiatry, University of Social Welfare and Rehabilitation Sciences, Tehran, Iran. \\ 2. Isfahan Psychiatric Hospital, Isfahan, Iran. \\ 3. Substance Abuse and Dependence Research Center, University of Social Welfare and Rehabilitation Sciences, Tehran, Iran. \\ 3. Psychiatry and Behavioral Sciences Research Center, Department of Psychiatry, Mashhad University of Medical Sciences, Mashhad, Iran.
}

Cittaton: Samiei, M., Hedayati, K., Mirabzadeh Ardekani, A., Dolatshahi, B., Daneshmand, R., \& Samadi, R. (2016). Obsessive-compulsive disorder in hospitalized patients with schizophrenia . Basic and Clinical Neuroscience, 7(4), 323-330. http://dx.crossref.org/10.15412/J.BCN.03070405

: http://dx.crossref.org/10.15412/J.BCN.03070405

Article info:

Received: 13 March 2016 First Revision: 10 April 2016 Accepted: 20 August 2016
Key Words:

Schizophrenia, Obsessive-compulsive symptoms, Iran, Persian Gulf

\begin{abstract}
A B S T RA C T
Introduction: Comorbid obsessive-compulsive disorder (OCD) has been reported among patients with schizophrenia in other countries. But, the literature is not well-documented on this issue in Iran (Persia). The present study aimed to investigate the prevalence and severity of OCD and some of its related factors in a group of patients with schizophrenia in Iran.

Methods: This is a cross-sectional study. A total of 150 hospitalized patients with schizophrenia were recruited at Razi Psychiatric Hospital in Tehran, Iran. Demographic and clinical checklists, as well as the Yale-Brown obsessive-compulsive scale (Y-BOCS), scale for assessment of negative symptoms (SANS), and scale for assessment of positive symptoms (SAPS) were administered to collect data. OCD was the dependent variable and independent variables included age, sex, severity of positive and negative symptoms, duration of schizophrenic disorder, the number of hospitalizations, and antipsychotic medications administered to them. Data were analyzed by analysis of variance (ANOVA), Chi-square, and T-test.
\end{abstract}

Results: Overall, $31.3 \%$ of patients had OCD with an average severity of $12.81(\mathrm{SD}=10.27)$. The prevalence of OCD was not affected by the number of psychiatric hospitalizations for schizophrenia or the duration of schizophrenic disorder. The severity of OCD significantly reduced as the duration of schizophrenia and the severity of negative symptoms increased.

Conclusion: OCD was found among a considerable proportion of the study sample. OCD may be associated with exacerbating schizophrenic symptoms. Therefore, psychiatrists should consider the simultaneous treatment of OCD and schizophrenia. Further studies are suggested in this issue.

\section{Introduction}

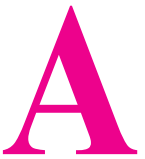

bout a century ago, obsessive-compulsive symptoms (OCSs) were identified. In recent decades, interest in conducting research on OCSs has increased because
OCSs exacerbate during psychosis treatment with atypical antipsychotic medications (Khullar Chue, \& Tibbo, 2001).

Patients with comorbid obsessive-compulsive disorder (OCD) and schizophrenia may represent a special category with special treatment needs. Diagnosis of OCSs

* Corresponding Author:

Reza Daneshmand, MD

Address: Department of Psychiatry, Psychiatry and Behavioral Sciences Research Center, Mashhad University of Medical Sciences, Mashhad, Iran. Tel: +98 (21) $22180095 \quad$ Fax: +98 (21) 22180095

E-mail: daneshmand74@yahoo.com 
in patients with schizophrenia is essentially important in diagnosing and implementing appropriate therapies. People diagnosed with the problem of obsessive-compulsive disorder (OCD) and schizophrenia are likely to be a specific group with specific psychiatric needs.

Diagnosis of OCSs in people diagnosed with schizophrenia is primarily crucial because this issue can help with the provision of appropriate psychiatric therapies for this group. It should be noted that similarities in the neural structures of people diagnosed with OCD and schizophrenia may explain why similar symptoms are seen in these subgroups (Adler \& Strakowski, 2003; Stein, 2002). Some brain pathways which are impacted by these illnesses include basal ganglia, anterior cingulate cortex, and orbitofrontal cortex (Graybiel \& Ruch, 2000). In schizophrenia, the dorsolateral prefrontal cortex circuit contains anatomic substrates similar to those of the OCD orbitofrontal circuit (Tibbo \& Warneke, 1999). Thus, the specific neuroanatomic sites identified by structural and functional neuroimaging studies in each of these disorders independently show considerable overlap in the implicated structures (Adler \& Strakowski, 2003), Some of these site are basal ganglia, thalamus, anterior cingulum, orbitofrontal cortex, and regions of the temporal cortex (Gross-Isseroff, Hermesh, Zohar, \& Weizman, 2003).

The results of most studies show a poorer clinical outcome and prognosis among schizophrenic patients with OCD (Frommhold, 2006). Poor general functioning, more disturbances in social behaviors (Lysaker, Lancaster, Nees, \& Davis, 2004), and more numbers of psychiatric hospitalizations are observed among schizophrenic patients with OCD (Saxena et al., 2002). In the study of Kayahan and colleagues (2005), the severity of OCD was correlated with positive symptoms of schizophrenia, but not with negative symptoms of schizophrenia, duration of schizophrenia, number of psychiatric hospitalizations, and the dosage of antipsychotic medications (Kayahan et al., 2005).

Psychiatric therapies of schizophrenia generally emphasize on the symptoms of schizophrenia, but comorbidities like OCD is commonly neglected and schizophrenic patients with OCD are not generally well-treated (Khan, Arshad, \& Ullah, 2004). There is a paucity of research on OCD among patients with schizophrenia in Iran while OCD requires important clinical and treatment implications. Lack of adequate attention to the prevalence of OCD as a comorbidity among patients with schizophrenia may result in poor treatment outcomes. Psychiatrists in Iran should seriously consider treating comorbidities such as OCD in the management of schizophrenia. To partly cover this gap, the current study investigated the prevalence and severity of OCD and some of its related factors among a group of hospitalized patients with schizophrenia at Razi Psychiatric Hospital in Tehran, Iran.

\section{Methods}

\subsection{Setting and participants}

The present study has a cross-sectional design. The study population included all hospitalized patients with schizophrenia at Razi Psychiatric Hospital in Tehran in 2011. The sample comprised 150 patients (127 males, 23 females) that were randomly selected. All patients who met DSMIV.TR criteria for schizophrenia were eligible to enter the study. Other inclusion criteria were at least 18 years old and cooperation with the researchers. Exclusion criteria included mental retardation, substance dependence or abuse except nicotine use, history of head stroke, having neurological disorders, lack of patient's cooperation, and taking antidepressant medications such as tricyclic antidepressants or serotonin reuptake inhibitors at the time of study.

\subsection{Research instruments}

Data were collected by the following instruments:

\subsubsection{A researcher-designed questionnaire}

A researcher-designed questionnaire was used to collect demographic and clinical characteristics of patients including age, gender, duration of schizophrenia, number of hospitalization, and their antipsychotic medications. Data were directly collected from patients or their relatives. The validity of the test was confirmed by reviewing previous studies and consulting with senior scholars in this field. The reliability of the questionnaire to meet the study aims was assessed by a 2-week pretest-posttest on 15 patients which showed a high reliability (94\%).

\subsubsection{Yale-Brown obsessive-compulsive scale}

Yale-Brown obsessive-compulsive scale (Y-BOCS) is a semi-structured clinical interview which was devised by Goodman and colleagues (1989) and measures the severity of OCSs in patients with schizophrenia (Goodman et al., 1989). Its score ranges between 0 and 40 . This scale is one of the most frequently used research tools, which has a standardized structure (Mcgawan \& Mctlroy, 2005). Previous studies in Iran show high reliability of applying this scale for Iranian patients (Mohammad-Khani, 1991). The validity of the tests was confirmed by reviewing pre- 
vious studies and consulting with senior scholars in this field. The reliability of Y-BOCS was assessed for this study by implementing a 2-week pretest-posttest on 15 patients which showed a high reliability (93\%).

\subsubsection{Scale for assessment of negative symptoms}

Scale for assessment of negative symptoms (SANS) was devised by Andreason in 1983 and has 24 items with high reliability which measures the severity of negative symptoms in patients with schizophrenia (Haan et al., 2006). Its score ranges between 0 and 120 . The validity of the test was confirmed by reviewing previous studies and consulting with senior scholars in this field. The reliability of SANS was assessed for this study by implementing a 2-week pretestposttest on 15 patients and a show a high reliability (95\%).

\subsubsection{Scale for assessment of positive symptoms}

Scale for assessment of positive symptoms (SAPS) has 35 items and its score ranges between 0 and 165 . This scale is one of the most frequently used research tools that has been revised by Andreason (1983) and has a high reliability (Bell, Milstein, Goulet, Lysaker, \& Cicchetti, 1992). The validity of the tests was confirmed by reviewing previous studies and consulting with senior scholars in this field. The reliability of SAPS was assessed for this study by implementing a 2-week pretest-posttest on 15 patients and a show a high reliability $(92 \%)$.

\subsection{Data analysis}

Data were analyzed by descriptive statistics, including mean, standard deviation, variance, Pearson correlation and inferential statistics, including analysis of variance (ANOVA), Chi-square test $\left(\mathrm{X}^{2}\right)$ and t-test by SPSS version 11 .

\subsection{Ethical considerations}

The protocol of the study was approved by the Ethics Committee of University of Welfare Sciences and Rehabilitation in Tehran, Iran. Oral and written consents were obtained from the participants who were assured of the confidentiality of the interviews. They were also assured that non-participation in the study would not affect their treatment procedure.

\section{Results}

The mean age of the participants was $39.5(\mathrm{SD}=10.67)$ years. The mean age of the men was $39(\mathrm{SD}=10.3)$ years while the mean age of the women was $38(\mathrm{SD}=9.4)$ years. Most of the patients (45\%) were married while the others were single (32\%) or divorced (23\%). All patients had less than 12 years of education. Mean duration of schizophrenia disorder among the patients was $12.1(\mathrm{SD}=6.56)$ years. Lifetime mean number of psychiatric hospitalization for schizophrenia was 6.01( $\mathrm{SD}=3.71)$ years. The mean score of the severity of positive symptoms of schizophrenia was $59.21(\mathrm{SD}=5.76)$ and of the negative symptoms was 49.04( $\mathrm{SD}=17.78)$. The mean score of the severity of OCD was $12.81(\mathrm{SD}=10.27)$. The results of clinical interviews (based on DSM-IV.TR) with patients showed that $31.3 \%$ of the patients had OCD (Table 1). Further data analyses (based on Y-BOCS) showed that 34\% had minor OCD

Table 1. The prevalence of OCD with respect to the number of hospitalization $(n=150)$.

\begin{tabular}{|c|c|c|c|c|}
\hline \multirow{2}{*}{ Number of psychiatric hospitalization } & OCD & OCD & \multirow{2}{*}{ Total } & \multirow{2}{*}{ P-value } \\
\cline { 2 - 5 } & Positive & Negative & 11 & 0.123 \\
\hline \multirow{2}{*}{$1-2$} & 3 & 8 & 100 & 0.05 \\
\cline { 2 - 5 } & 27.3 & 72.7 & 59 & 0.05 \\
\hline \multirow{2}{*}{$3-4$} & 17 & 42 & 100 & 0.05 \\
\cline { 2 - 5 } & 28.8 & 22 & 32 & 0.05 \\
\hline \multirow{2}{*}{$5-6$} & 10 & 68.8 & 100 & 0.05 \\
\cline { 2 - 5 } & 31.3 & 8 & 14 & 0.127 \\
\hline \multirow{2}{*}{$7-8$} & 6 & 57.1 & 8 & 0.125 \\
\hline \multirow{2}{*}{$9-10$} & 42.9 & 5 & 100 & 0.141 \\
\hline \multirow{2}{*}{$>10$} & 3 & 72.5 & 26 & 0.05 \\
\hline \multirow{2}{*}{ Total } & 37.5 & 18 & 100 & 0.05 \\
\hline & 30.8 & 69.2 & 150 & 0 \\
\hline
\end{tabular}


Table 2. Severity of OCD with respect to the number of hospitalization $(n=150)$.

\begin{tabular}{|c|c|c|c|}
\hline Variable & Sample & \multicolumn{2}{|c|}{ Severity of OCSs } \\
\hline Number of hospitalization & No. & Mean & 6.66 \\
\hline $1-2$ & 3 & 18.67 & 15.07 \\
\hline $3-4$ & 17 & 15.29 & 4.32 \\
\hline $5-6$ & 10 & 11.00 & 8.85 \\
\hline $7-8$ & 6 & 9.33 & 2.08 \\
\hline $9-10$ & 3 & 14.67 & 4.69 \\
\hline$>10$ & 8 & 9.50 & 10.27 \\
\hline
\end{tabular}

$\mathrm{F}=0.758, \mathrm{P}=0.585$.

NEUR:SCIENCE

Table 3. Prevalence of OCD symptoms with respect to duration of schizophrenia ( $n=150)$.

\begin{tabular}{|c|c|c|c|c|}
\hline \multirow{2}{*}{$\begin{array}{c}\text { Variable } \\
\text { Duration of Schizophrenia (Year) }\end{array}$} & \multicolumn{4}{|c|}{ Symptoms of OCD } \\
\hline & Number/Percentage & Negative & Positive & Total \\
\hline \multirow{2}{*}{$<6$} & $\mathrm{n}$ & 5 & 14 & 19 \\
\hline & $\%$ & 26.3 & 73.7 & 100 \\
\hline \multirow{2}{*}{$6-10$} & $\mathrm{n}$ & 15 & 33 & 48 \\
\hline & $\%$ & 31.3 & 68.8 & 100 \\
\hline \multirow{2}{*}{$11-15$} & $\mathrm{n}$ & 13 & 28 & 41 \\
\hline & $\%$ & 31.7 & 68.3 & 100 \\
\hline \multirow{2}{*}{$16-20$} & $\mathrm{n}$ & 12 & 13 & 25 \\
\hline & $\%$ & 48.0 & 52.0 & 100 \\
\hline \multirow{2}{*}{$>21$} & $\mathrm{n}$ & 2 & 15 & 17 \\
\hline & $\%$ & 11.8 & 88.2 & 100 \\
\hline \multirow{2}{*}{ Total } & $\mathrm{n}$ & 47 & 103 & 150 \\
\hline & $\%$ & 31.3 & 68.7 & 100 \\
\hline
\end{tabular}

$\mathrm{P}>0.05$ is significant.

NEUR:SCIENCE

Table 4. Severity of OCD negative symptoms with respect to duration of Schizophrenia $(n=150)$.

\begin{tabular}{|c|c|c|c|}
\hline Duration of schizophrenia (Year) & No. & Mean & SD \\
\hline$<6$ & 5 & 27.20 & 24.67 \\
\hline $6-10$ & 15 & 11.47 & 4.93 \\
\hline $11-15$ & 13 & 12.69 & 6.22 \\
\hline $16-20$ & 12 & 10 & 5.36 \\
\hline$>21$ & 2 & 4.50 & 2.12 \\
\hline Total & 47 & 12.81 & 10.27 \\
\hline
\end{tabular}

$\mathrm{F}=3.821, \mathrm{P}=0.01$

NEUR:SCIENCE 
(score of less than 10), 44.7\% had moderate OCD (scores $10-15$ ), 17\% had high OCD (scores 16-25), and 4.3\% had very high OCD (scores more than 25).

The prevalence and the severity of OCD in the patients had no significant relationship with the number of psychiatric hospitalization for schizophrenia. The prevalence of OCD in the patients had also no significant relationship with the duration of schizophrenia (Table 2) but had a significant relationship with the severity of OCD (Table 3 ). This issue showed that the shorter duration of schizophrenia was associated with more severe cases of OCSs.

Mean score of the severity of OCD had no significant relationship with the positive symptoms of schizophrenia (Table 3). There was a significant relationship between OCD and the severity of negative symptoms of schizophrenia (Table 4). This issue showed that the more severe the negative symptoms of schizophrenia, the more severe will be OCD.

\section{Discussion}

Psychiatric comorbidity such as OCD could be a health concern among hospitalized patients with schizophrenia in Iran and the simultaneous treatment is an inventible part of schizophrenia treatment. The present study showed that OCD was prevalent in a group of hospitalized patients with schizophrenia at Razi Psychiatric Hospital in Tehran. The present study is one of the few studies in Iran that has been specifically conducted on the prevalence and severity of OCD among Iranian schizophrenic patients and its related factors. The study findings showed that $31 \%$ of the participants were diagnosed with OCD, which is similar to other studies about the prevalence of OCD among schizophrenic patients in developed countries (Byerly, Goodman, Acholonu, Bungno, \& Rush, 2005; Kayahan et al., 2005.

Research studies on neuroimaging indicate that there could be a particular pattern of brain dysfunction in this comorbid condition. Aoyama et al. (2000) conducted a MRI study on patients with schizophrenia and OCD and found out that left hippocampus was primarily smaller in this group compared with the patients who were only diagnosed with schizophrenia. An inverse correlation was detected between illness duration and the size of the frontal brain lobe in the group with comorbid diagnosis of schizophrenia and OCD (Aoyama et al., 2000).

The prevalence of OCD among our patients has important clinical and treatment implications and should be considered during treatment of schizophrenia. Further studies on the brain regions involved in this issue among our patients can also contribute to better diagnosis and treatment of this disorder. Differences in the study findings about the prevalence and severity of OCD among patients with schizophrenia in Iran may be related to many factors, including difficulty in diagnosing the differences between obsessive symptoms and compulsive symptoms from psychotic symptoms, the duration of taking antipsychotic medications, differences in dosage used, different prescribed antipsychotic medications, differences in demographic characteristics, applying different scales for determining OCD, and different stages (the acute stages or chronic stage) of schizophrenia (GalvezBuccollini et al., 2004).

The present study findings are consistent with some studies (Berman et al., 1999) which showed no relation between the number of psychiatric hospitalization for schizophrenia with the severity of OCD. This finding may be related to the independence of OCD from the signs and symptoms of schizophrenia disorder (Bell et al., 1992). Differences in the severity of OCD in schizophrenic patients in this study were consistent with the similar studies (Berman et al., 1999). The study findings showed that no relationship exists between the prevalence of OCD and the duration of schizophrenia, while some studies have shown that the severity of OCD decreases with the duration of schizophrenia. Therefore, OCD may be similar to positive symptoms of schizophrenia in that its severity decreases with time (Kayahan et al., 2005).

It is also possible that the severity of OCD decreases under the influence of taking antipsychotic medications, however, this issue needs further research. The study findings showed a negative correlation between negative symptoms of schizophrenia and OCD which is inconsistent with other studies. In some previous studies, OCD had no relationship with positive and negative symptoms of schizophrenia (Byerlyet al., 2005; Poyurovsky et al., 2000), but in some other studies, OCD had positive relationship with negative symptoms of schizophrenia (Tibbo \& Warneke, 1999; Poyurovsky, Bergman, Shoshani, Schneidman, \& Weizman, 1998). This difference could be due to differences in sample size, duration of treatment, taking antipsychotic medications with different dosages. However, this topic still needs further research.

Based on the study, OCD and schizophrenia among our subjects may indicate the presence of comorbid disorders which could have influence on each other. However, more studies are still required on this issue because some studies show that the prevalences of obsessive-compulsive disorder and schizophrenia are 2\%-3\% and 1\%-1.5\%, respectively (Sadock \& Sadock, 2007). It should be noted that OCD may be a minor part of positive symptoms of schizophrenia. The reduction in severity of OCD after increasing the positive symptoms of schizophrenia and the 
significant relationship between them may indicate the same physiological and brain bases between OCD and the positive symptoms of schizophrenia. However, it still needs further research on the involvements of common brain regions and neurotransmitter systems.

There is very limited neurobiological research focusing specifically on the overlap between OCD and neuroimaging; however, fMRI studies may have distinguished specific brain structural abnormalities unique to this putative subtype. Some abnormalities in the brain structure are unique to this pervasive subtype (OCD and schizophrenia comorbidity). Although studies on neuropsychological testing have not detected a unique form of impairment in this group, it has been primarily shown that there is more impairment in this group compared to people who have only diagnosed with schizophrenia or OCD. This issue suggests a strong relationship between these two illnesses. More neurobiological and neurocognitive studies are required to investigate the nature of these psychiatric illnesses and their similarities in future.

The current study had also some limitations. First of all, there was no control group, and the number of female participants was not adequate. Further studies are suggested with adding a control group and representative numbers of female samples. Comorbid such as OCD is rarely considered for simultaneous treatment among patients with schizophrenia in Iran. This topic should be considered more in future research studies.

\section{Acknowledgments}

This study is self-funded. The authors would also like to thank all participants and hospital staff who contributed in the study.

\section{Conflict of Interests}

The authors declared no conflict of interest.

\section{References}

Aoyama, F., Iida, J., Inoue, M., Iwasaka, H., Sakiyama, S., Hata, K., et al. (2000). Brain imaging in childhood- and adolescentonset schizophrenia associated with obsessive-compulsive symptoms. Acta Psychiatrica Scandinavica, 102(1), 32-37.

Adler, C. M., \& Strakowski, S. M. (2003). Boundaries of schizophrenia. Psychiatric Clinics of North America, 26(1), 1-23.

Bell, M. D., Milstein, R. M., Goulet, J. B., Lysaker, P., \& Cicchetti, D. (1992). The positive and negative syndrome scale and brief psychiatry rating scale: reliability and predictive validity. Journal of Nervous and Mental Disease, 180(11), 723-28.

Byerly, M., Goodman, W., Acholonu, W., Bungno, R., \& Rush, A. J. (2005). Obsessive compulsive symptoms in schizophrenia: Frequency and clinical features. Schizophrenia Research, 76(2-3), 309-16

Berman, I., Chang, H. H., \& Klegon, D. A. (1999). Obsessive compulsive disorder in schizophrenia: neuropsychological perspectives. Psychiatric Annals, 29(9), 525-28.

Berman, I. (2001). Obsessive compulsive disorder in schizophrenia. Psychiatric Times, 18(2), 373-79.

Frommhold, K. (2006). Obsessive-compulsive disorder and schizophrenia: a critical review. Fortschritte der Neurologie Psychiatrie, $7(1), 74-83$.

Galvez-Buccollini, J. A., Fiestas, F., Herrera, P., Vega-Dienstmaier, J. M., Guimas, B., \& Mazzotti, G. (2004). Obsessive compulsive symptoms in schizophrenia during treatment with clozapine an conventional antipsychotic drugs. Actas Españolas de Psiquiatría, 32(4), 211-15.

Goodman, W. K., \& Price, L. H., Rasmussen, S. A., \& Mazure, C., Delgado, P., Heninger, G. R., et al. (1989). The Yale-Brown obsessive-compulsive scale: development, use, reliability, and validity. Archives of General Psychiatry, 46(11), 1006-016.

Gross-Isseroff, R., Hermesh, H., Zohar, J., \& Weizman, A. (2003). Neuroimaging communality between schizophrenia and obsessive compulsive disorder: a putative basis for schizoobsessive disorder? World Journal of Biological Psychiatry, 4(3), 129-34.

Graybiel, A. M., \& Rauch, S. L. (2000). Toward a neurobiology of obsessive-compulsive disorder. Neuron, 28(2), 343-47.

Haan, L., Hoogeboom, B., Beuk, N., Wouters, L., Dingemans, P. \& Linszen, D. H. (2006). Reliability and validity of the YaleBrown obsessive-compulsive scale in Schizophrenia patients. Psychopharmacology Bulletin, 39(1), 25-30.

Khan, M. N., Arshad, N., \& Ullah, N. (2004). Treatment outcome of Schizophrenia co-morbid with obsessive-compulsive disorder. Journal of College of Physicians and Surgeons Pakistan, 14(4), 234-36.

Khullar, A., Chue, P., \& Tibbo, P. (2001). Quetiapine and obsessive-compulsive symptoms (OCS): case report and review of atypical antipsychotic-induced OCS. Journal of Psychiatry \& Neuroscience, 26(1),55-59.

Kayahan, B., Ozturk, O., Veznedaroglu, B., \& Eraslan, D. (2005). Obsessive compulsive symptoms in schizophrenia: prevalence and clinical correlates. Psychiatry and Clinical Neurosciences, 59(3), 291-95.

Lysaker, P. H., Lancaster, S. R., Nees, M. A., \& Davis, L. W (2004). Patterns of obsessive compulsive symptoms and socia function in schizophrenia. Psychiatry Research, 125(2), 139-46.

Mcgawan, I. W., \& Mctlroy, J. (2005). Significance of obsession and compulsion in schizophrenia. Journal of Clinical Psychiatry, $24(2), 1-8$.

Mohammad-Khani, P. (1991). [Coping strategies for handling the stress and symptoms in patients with obsessive-compulsive disorder (Persian)] (MA thesis). Tehran: Tehran University of Medical Sciences. 
Poyurovsky, M., Hramenkov, S., Isakov, V., Rauchverger, B., Modai, I., Schneidman, M., et al. (2000). Obsessive compulsive disorder in hospitalized patients with chronic Schizophrenia. Psychiatry Research, 102(1), 49-57.

Poyurovsky, M., Bergman, Y., Shoshani, D., Schneidman, M., \& Weizman, A. (1998). Emergence of obsessive compulsive symptoms and tics during clozapine withdrawal. Clinical Neuropharmacology, 21(2), 97-100.

Saxena, S., Maidment, K. M., Vapnik, T., Golden, G., Rishwain, T., Rosen, R. M., et al. (2002). Obsessive-compulsive hoarding: symptom severity and response to multimodal treatment. Journal of Clinical Psychiatry, 63(1), 21-27.

Stein, D. J. (2002). Obsessive-compulsive disorder. Lancet, 360(9930), 397-405.

Sadock, B. J., \& Sadock, V. A. (2007). Synopsis of psychiatry (10th ed.). Philadelphia: Lippincott William \& Wilkins Publication.

Tibbo, P., \& Warneke, L. (1999). Obsessive-compulsive disorder in schizophrenia: epidemiologic and biologic overlap. Journal of Psychiatry \& Neuroscience, 24(1),15-24. 
